\title{
Bousfield Localization Functors and Hopkins' Chromatic Splitting Conjecture
}

\author{
MARK HOVEY
}

July 1993

\section{Introduction}

This paper arose from attempting to understand Bousfield localization functors in stable homotopy theory. All spectra will be $p$-local for a prime $p$ throughout this paper. Recall that if $E$ is a spectrum, a spectrum $X$ is $E$-acyclic if $E \wedge X$ is null. A spectrum is $E$-local if every map from an $E$-acyclic spectrum to it is null. A map $X \rightarrow Y$ is an $E$-equivalence if it induces an isomorphism on $E_{*}$, or equivalently, if the fibre is $E$-acyclic. In [Bou79], Bousfield shows that there is a functor called $E$-localization, which takes a spectrum $X$ to an $E$-local spectrum $L_{E} X$, and a natural transformation $X \rightarrow L_{E} X$ which is an $E$-isomorphism. Studying $L_{E} X$ is studying that part of homotopy theory which $E$ sees.

These localization functors have been very important in homotopy theory. Ravenel [Rav84] showed, among other things, that finite spectra are local with respect to the wedge of all the Morava K-theories $\bigvee_{n<\infty} K(n)$. This gave a conceptual proof of the fact that there are no non-trivial maps from the EilenbergMacLane spectrum $H \mathbf{F}_{p}$ to a finite spectrum $X$.

Hopkins and Ravenel later extended this to the chromatic convergence theorem [Rav92]. If we denote, as usual, the localization with respect to the first $n+1$ Morava K-theories $K(0) \vee \cdots \vee K(n)$ by $L_{n}$, the chromatic convergence theorem says that for finite $X$, the tower $\ldots \pi_{i} L_{n} X \rightarrow \pi_{i} L_{n-1} X \ldots \rightarrow \pi_{i} L_{0} X$ is pro-isomorphic to the constant tower $\left\{\pi_{i} X\right\}$. In particular, $X$ is the inverse limit of the $L_{n} X$.

1991 Mathematics Subject Classification. 55P42, 55P60, 55N22, 55N20.

This paper is in final form and no version of it will be submitted elsewhere. 
The major result of this paper is that finite torsion spectra are local with respect to any infinite wedge of Morava K-theories $\bigvee_{i<\infty} K\left(n_{i}\right)$. This has several interesting corollaries. For example, it implies that there are no maps from the Johnson-Wilson spectra $B P\langle n\rangle$ to a finite spectrum. It also implies that if $E$ is a ring spectrum which detects all finite spectra, so that $E_{*}(X) \neq 0$ if $X$ finite, then $L_{E} X$ is either $X$ or $X_{p}$, the $p$-completion of $X$, for finite $X$. This in turn implies that the only smashing localization which detects all finite complexes is the identity functor.

In order to prove that finite torsion spectra are $\bigvee_{i} K\left(n_{i}\right)$ local, I show that $B P_{p}$ is a wedge summand of $\prod_{i} L_{K\left(n_{i}\right)} B P_{p}$. This is saying that one does not have to reassemble the chromatic pieces of $B P_{p}$ into an inverse limit to recover the homotopy theory of $B P_{p}$. This result is a $B P$ analogue of the chromatic splitting conjecture of Hopkins. I will describe this conjecture in Section 4 , but for now suffice it to say that the conjecture is that $L_{n-1} X_{p}$ is a wedge summand in $L_{n-1} L_{K(n)} X_{p}$. The chromatic splitting conjecture is actually stronger than that, for it also explains how this splitting occurs, but most of the corollaries I draw from the chromatic splitting conjecture only need the splitting itself. One corollary of the chromatic splitting conjecture would be that, for finite $X, X_{p}$ is a wedge summand of $\prod_{i} L_{K\left(n_{i}\right)} X_{p}$, explaining how my result is a $B P$ analogue of the chromatic splitting conjecture. I do not know if $L_{n-1} B P_{p}$ is a wedge summand of $L_{n-1} L_{K(n)} B P_{p}$.

In the first two sections of this paper, I describe some other results about Bousfield localization functors, this time with respect to spectra $E$ which kill a finite spectrum. The pedigree of these results is somewhat confusing. Almost all of the results in Sections 1 and 2 have been known to Hopkins for some time. Others may have known them as well, but they have not appeared in print before. I feel that they warrant a larger audience. In addition, I discovered many of these results independently, and there are a couple of new results as well. For example, I show that $L_{K(n)}$ is a minimal localization functor. That is, if the natural transformation $X \rightarrow L_{E} X$ factors through $L_{K(n)} X$, then $L_{E} X$ is either the zero functor or is $L_{K(n)} X$ itself. I also provide some new examples of smashing localizations.

The last section of the paper discusses the consequences of the chromatic splitting conjecture on the homotopy groups of $L_{n} S^{0}$. We show that, given the chromatic splitting conjecture, the divisible summands in $\pi_{*} L_{n} S^{0}$ for $n \geq 1$ can be determined. There are $3^{n-1}$ of them, with $2^{n-1}$ of them occuring in dimension $-2 n$, and the others spread out from dimension $-2 n-1$ to dimension $-n^{2}-1$. This therefore explains part of the Shimomura-Yabe calculation of $\pi_{*} L_{2} S^{0}$ for $p>3$ in $[\mathbf{S h}-\mathbf{Y}]$.

This paper is written in the homotopy category of $p$-local spectra. In particular, '=' is equality in the homotopy category, namely homotopy equivalence. Similarly, I will often write that a map or spectrum is 0 , by which I mean that it is null-homotopic or contractible. 
I would like to thank Mike Hopkins for sharing his ideas so freely. I thank Hal Sadofsky for hundreds of discussions on matters related to this paper. I also thank David Johnson for helpful discussions, and Paul Eakin and Avinash Sathaye for convincing me that my original ideas about infinite abelian groups were too naive.

\section{Spectra with finite acyclics}

Before describing the results of this section, I need to recall the definition of the Bousfield class of a spectrum [Bou79].

Definition 1.1. Two spectra $E$ and $F$ are Bousfield equivalent if, given any spectrum $X$,

$$
E \wedge X=0 \text { if and only if } F \wedge X=0 .
$$

Denote the equivalence class of $E$ by $\langle E\rangle$. Define $\langle E\rangle \leq\langle F\rangle$ if and only if $F \wedge X=0$ implies $E \wedge X=0$. Define

$$
\langle E\rangle \wedge\langle F\rangle=\langle E \wedge F\rangle
$$

and

$$
\langle E\rangle \vee\langle F\rangle=\langle E \vee F\rangle \text {. }
$$

There is a minimal Bousfield class $\langle *\rangle$, which we will often denote by 0 , and a maximal Bousfield class $\left\langle S^{0}\right\rangle$. I remind the reader that it is perfectly possible to have $\langle E\rangle \leq\langle F\rangle$ while nonetheless $\langle E \wedge F\rangle=0$.

In this section we investigate Bousfield classes of spectra $E$ which have finite acyclics, i.e. there is some finite $X$ with $E \wedge X=0$. Highlights of this section include the minimality of the Bousfield class of $K(n)$ (Corollary 1.7) and the new examples of smashing localizations (Proposition 1.5). We also show that every $B P$-module spectrum with finite acyclics has the Bousfield class of a wedge of Morava K-theories, and that a $v_{n}$-periodic Landweber exact spectrum has the same Bousfield class as $E(n)$.

First, we need to recall some corollaries of the nilpotence theorem [DHS, HS]. Recall that a finite spectrum $X$ has type $n$ if $K(i)_{*}(X)=0$ for $i<n$ but $K(n)_{*}(X) \neq 0$. Every finite spectrum is of some finite type, and the periodicity theorem of J. Smith, written up in [Rav92], says that there is a spectrum of type $n$ for all $n$. Let $\mathcal{C}_{n}$ denote the class of all finite spectra of type at least $n$. Then [HS] any nonempty collection of finite spectra that is closed under cofibrations and retracts is some $\mathcal{C}_{n}$.

LEMma 1.2 (Hopkins-Smith). All finite spectra of type $n$ have the same Bousfield class, which we denote $F(n)$.

Proof. This is an easy application of the nilpotence theorem. Given an $X$ of type $n$, let $\mathcal{C}$ consist of all finite spectra $Y$ such that $\langle Y\rangle \leq\langle X\rangle$. It is easy to see that $\mathcal{C}$ is closed under retracts, cofibrations, and suspensions, so must be a 
$\mathcal{C}_{k}$ for some $k$. Since $X \in \mathcal{C}, \mathcal{C} \subseteq \mathcal{C}_{n}$. In particular, if $Y$ is type $n,\langle Y\rangle \leq\langle X\rangle$. Interchanging $X$ and $Y$ completes the proof.

A spectrum $X$ in $\mathcal{C}_{n}$ has a $v_{n}$ self-map, that is, a map inducing an isomorphism on $K(n)_{*}(X)$ [HS]. Any two such become equal upon iterating them enough times, so that there is a well-defined telescope $T e l_{n}(X)$. Tel $l_{n}$ is actually an exact functor on the category of finite spectra with $v_{n}$ self maps. This follows from the fact that a map between two such finite spectra will commute with large enough iterates of the $v_{n}$ self maps. By following a similar line of proof as in the above lemma, we get

Lemma 1.3. The telescopes of finite spectra of type $n$ all have the same Bousfield class, which we denote Tel(n).

This lemma was also known to Hopkins and Smith, and it appears in [MS] as well.

Recall the lemma of [Rav84]: if $f$ is a self-map of $X$ and $\operatorname{Tel}(X)$ is its telescope and $Y$ its cofibre, then $\langle X\rangle=\langle\operatorname{Tel}(X)\rangle \vee\langle Y\rangle$. Applying this repeatedly using $v_{n}$ self maps, we get

$$
\left\langle S^{0}\right\rangle=\langle\operatorname{Tel}(0)\rangle \vee \cdots \vee\langle\operatorname{Tel}(n)\rangle \vee\langle F(n+1)\rangle .
$$

This decomposition is the key to most of our results in this section. Note that it is orthogonal, in the sense that $\operatorname{Tel}(m) \wedge \operatorname{Tel}(n)=\operatorname{Tel}(m) \wedge F(n)=0$ if $m<n$. This is proven in [MS].

Given any spectrum $E$, let

$$
\mathrm{FA}(E)=\{X \mid X \text { is finite and } E \wedge X=0\} .
$$

In this section, we will discuss spectra which have finite acyclics, so that we assume $\mathrm{FA}(E) \neq\{*\}$. It is easy to see that $\mathrm{FA}(E)$ is closed under cofibrations and retracts, so it must be $\mathcal{C}_{n+1}$ for some $n$. We then have the following observation.

Lemma 1.4. If $F A(E)=\mathcal{C}_{n+1}$, then

$$
\langle E\rangle=\langle E \wedge \operatorname{Tel}(0)\rangle \vee \cdots \vee\langle E \wedge \operatorname{Tel}(n)\rangle .
$$

In particular, $\langle\operatorname{Tel}(0) \vee \cdots \vee \operatorname{Tel}(n)\rangle$ is the largest Bousfield class with finite acyclics $\mathcal{C}_{n+1}$, and therefore localization with respect to it, denoted $L_{n}^{f}$, is smashing.

Proof. Just smash equation (1) with $E$. To see $L_{n}^{f}$ is smashing, note that for any spectrum $E, \mathrm{FA}\left(L_{E} S^{0}\right)=\mathrm{FA}(E)$. Thus,

$$
\left\langle L_{n}^{f} S^{0}\right\rangle \leq\langle\operatorname{Tel}(0) \vee \cdots \vee \operatorname{Tel}(n)\rangle .
$$

This implies by Prop. 1.27 of [Rav84] that $L_{n}^{f}$ is smashing. 
$L_{n}^{f}$ has been investigated by many authors [Bou92, MS, Mil, Rav92a]. All of them noticed that it is smashing, though I think this is the most transparent proof. The telescope conjecture is usually stated as saying that if $X$ is type $n$ then $L_{K(n)} X=\operatorname{Tel}(X)$. This is equivalent to $\langle\operatorname{Tel}(n)\rangle=\langle K(n)\rangle$, and also to $L_{n}^{f}=L_{n}$. (For details, see [MS] or one of the other cited papers above.) This conjecture is now known to be false for $n=2[$ Rav92b, MRS], and is presumed to be false for larger $n$ as well.

As an amusing example of what the failure of the telescope conjecture means, we include the following proposition.

Proposition 1.5. Localization with respect to

$$
\operatorname{Tel}(0) \vee \cdots \vee \operatorname{Tel}(m) \vee K(m+1) \vee \cdots \vee K(n)
$$

is smashing.

Proof. Call this localization functor $L_{m, n}$. We have that

$$
\langle\operatorname{Tel}(0) \vee \cdots \vee \operatorname{Tel}(m)\rangle \leq\left\langle L_{m, n} S^{0}\right\rangle \leq\langle\operatorname{Tel}(0) \vee \cdots \vee \operatorname{Tel}(n)\rangle .
$$

We need to show that $\left\langle L_{m, n} S^{0} \wedge \operatorname{Tel}(i)\right\rangle=\langle K(i)\rangle$ if $m<i \leq n$. Note that

$$
\left\langle L_{m, n} S^{0} \wedge \operatorname{Tel}(i)\right\rangle=\left\langle L_{m, n} S^{0} \wedge F(i) \wedge \operatorname{Tel}(i)\right\rangle=\left\langle L_{m, n} F(i) \wedge \operatorname{Tel}(i)\right\rangle .
$$

But $F(i)$ is $\operatorname{Tel}(0) \vee \cdots \vee T e l(m)$ acyclic, so $L_{m, n} F(i)=L_{n} F(i)$. Since $L_{n}$ is smashing [Rav92], $\left\langle L_{n} F(i)\right\rangle=\langle K(i) \vee \cdots \vee K(n)\rangle$, and the result follows.

It is an old problem of Bousfield's to classify all smashing localization functors. We address another part of this problem in Section 3.

To measure the extent to which the telescope conjecture fails, note that there is a natural map $L_{n}^{f} X \rightarrow L_{n} X$. Let $A_{n} X$ be the fibre of this map. Note that if $X$ is type $n$, this is also the fiber of the map $\operatorname{Tel}(X) \rightarrow L_{K(n)}(X)$, for then $L_{n}^{f}(X)=\operatorname{Tel}(X)([\mathbf{M S}])$, and $L_{n} X=L_{K(n)}(X)$.

Proposition 1.6. If $X$ is finite and type $n$, the Bousfield class of $A_{n} X$ does not depend on $X$. We denote it $A(n)$. $\langle\operatorname{Tel}(n)\rangle=\langle K(n)\rangle \vee\langle A(n)\rangle$, and $A(n) \wedge$ $K(m)=0$ for all $m$.

Proof. First note that because $L_{n}^{f}$ and $L_{n}$ are both smashing (see [Rav92] for $L_{n}$ ), so is $A_{n}$. That is, $A_{n} X=A_{n} S^{0} \wedge X$ for all $X$. In particular, if $X$ and $Y$ are Bousfield equivalent, so are $A_{n} X$ and $A_{n} Y$. This shows that $\langle A(n)\rangle$ is well-defined. The map $L_{n}^{f} X \rightarrow L_{n} X$ is an isomorphism on $K(m)$ homology for all $m$, (and also on $B P$ homology as we will see below), so $A(n) \wedge K(m)=0$ for all $m$. If $X$ is type $n,\langle\operatorname{Tel}(X)\rangle=\langle\operatorname{Tel}(n)\rangle$ and $\left\langle L_{K(n)} X\right\rangle=\langle K(n)\rangle$, and it follows that $\langle\operatorname{Tel}(n)\rangle=\langle K(n)\rangle \vee\langle A(n)\rangle$.

$A(n)$ behaves very much like $M_{n} X$, the $n$th monochromatic layer, which is the fiber of $L_{n} X \rightarrow L_{n-1} X$. In particular, we have that $A(n) \wedge A(n)$ is homotopy equivalent to $A(n)$, and $L_{A(n)} A(n)$ is homotopy equivalent to $L_{A(n)} S^{0}$. 
Corollary 1.7. $\langle K(n)\rangle$ is a minimal Bousfield class. That is, if $\langle E\rangle<$ $\langle K(n)\rangle$, then $E$ is null.

Proof. Suppose $\langle E\rangle \leq\langle K(n)\rangle$. Then

$$
\langle E \wedge \operatorname{Tel}(m)\rangle \leq\langle K(n) \wedge \operatorname{Tel}(m)\rangle=0
$$

if $n \neq m$. Similarly, $\langle E \wedge F(n+1)\rangle \leq\langle K(n) \wedge F(n+1)\rangle=0$. Thus, from equation (1), we have that $\langle E\rangle=\langle E \wedge T(n)\rangle$. But, also

$$
\langle E \wedge A(n)\rangle \leq\langle K(n) \wedge A(n)\rangle=0,
$$

so by the preceeding proposition, we have $\langle E\rangle=\langle E \wedge K(n)\rangle$. Since $K(n)$ is a field spectrum, $E \wedge K(n)$ is a wedge of suspensions of $K(n)$, so there are only two possibilities for $\langle E \wedge K(n)\rangle, 0$ or $\langle K(n)\rangle$.

Note that the corresponding result is not true for the other field spectrum, $H \mathbf{F}_{p}$. Indeed, in the proof of Theorem 2.2 of [Rav84], Ravenel shows that $\langle Y\rangle\left\langle\left\langle H \mathbf{F}_{p}\right\rangle\right.$, where $Y$ denotes the Brown-Comanetz dual of $B P \wedge M(p)$.

A similar argument to the above shows that if $\langle E\rangle$ is less than or equal to some finite wedge of Morava K-theories, then $E$ must be Bousfield equivalent to a finite wedge of Morava K-theories. This says in particular that the chromatic tower is unrefineable. There is no localization functor $L_{E}$ that fits between $L_{n}$ and $L_{n-1}$.

In the light of this result and the failure of the telescope conjecture, one might ask if $A(n)$ is also a minimal Bousfield class. This would say that the telescope conjecture is not so badly wrong. I think this is likely to be true, but since I have no data, I will not be so bold as to conjecture it.

The following theorem will show that the telescope conjecture is true after smashing with $B P$. This has been known to Hopkins, Ravenel, and probably others, though it has not appeared before. First we need a lemma.

Lemma 1.8. Suppose $R$ is a ring spectrum and the unit map $S^{0} \stackrel{\eta}{\rightarrow} R$ factors through some spectrum $E$. Then $\langle E\rangle \geq\langle R\rangle$.

Proof. Since $R$ is a ring spectrum, the composite

$$
R=S^{0} \wedge R \stackrel{\eta \wedge 1}{\rightarrow} R \wedge R \rightarrow R
$$

is the identity. Since $\eta$ factors through $E$, the identity map on $R$ factors through $E \wedge R$. So if $E \wedge Z$ is null, so is $R \wedge Z$.

Recall that $P(n)$ is a $B P$-module spectrum whose homotopy is

$$
\pi_{*} P(n)=B P_{*} /\left(p, v_{1}, \ldots, v_{n-1}\right) .
$$

The first part of the following theorem is Ravenel's theorem 2.1(g) in [Rav84]. We reprove it so as to make the second part clearer. 
TheOREM 1.9 .

$$
\langle B P \wedge F(n)\rangle=\langle P(n)\rangle
$$

and

$$
\langle B P \wedge \operatorname{Tel}(n)\rangle=\langle K(n)\rangle .
$$

Proof. If there were a spectrum $V(n-1)$ with

$$
B P_{*} V(n-1)=B P_{*} /\left(p, v_{1}, \ldots, v_{n-1}\right),
$$

it would be type $n$ and we would have $B P \wedge V(n-1)=P(n)$, so the result would be obvious. In general, there are not such spectra, but there are appropriate substitutes $M\left(p^{i_{0}}, v_{1}^{i_{1}}, \ldots, v_{n-1}^{i_{n-1}}\right)$ constructed by Devinatz in [Dev]. These exist for sufficiently large $\left(i_{0}, i_{1}, \ldots, i_{n-1}\right)$, they are finite of type $n$, and they have the evident $B P$-homology. Furthermore, $B P \wedge M\left(p^{i_{0}}, v_{1}^{i_{1}}, \ldots, v_{n-1}^{i_{n-1}}\right)$ can be constructed from $P(n)$ using cofibre sequences, in the same way that the mod $p^{n}$ Moore space can be constructed from the $\bmod p$ Moore space. Therefore

$$
\left\langle B P \wedge M\left(p^{i_{0}}, v_{1}^{i_{1}}, \ldots, v_{n-1}^{i_{n-1}}\right)\right\rangle \leq\langle P(n)\rangle .
$$

Note that there is a natural map of $B P$-module spectra

$$
B P \wedge M\left(p^{i_{0}}, v_{1}^{i_{1}}, \ldots, v_{n-1}^{i_{n-1}}\right) \rightarrow P(n) .
$$

The unit map $S^{0} \rightarrow P(n)$ of the ring spectrum $P(n)$ factors through this map, so by the proceeding lemma,

$$
\left\langle B P \wedge M\left(p^{i_{0}}, v_{1}^{i_{1}}, \ldots, v_{n-1}^{i_{n-1}}\right)\right\rangle \geq\langle P(n)\rangle .
$$

It can actually be shown using a variant of the Landweber exact functor theorem and Lemma 2.13 of [Rav84] that $P(n)$ is a module spectrum over $B P \wedge$ $M\left(p^{i_{0}}, v_{1}^{i_{1}}, \ldots, v_{n-1}^{i_{n-1}}\right)$, but we do not need this.

To see that $\langle B P \wedge \operatorname{Tel}(n)\rangle=\langle K(n)\rangle$, we proceed similarly. A $v_{n}$ self map on $M\left(p^{i_{0}}, v_{1}^{i_{1}}, \ldots, v_{n-1}^{i_{n-1}}\right)$ induces multiplication by a power of $v_{n}$ on $B P$-homology, so

$$
B P \wedge \operatorname{Tel}\left(M\left(p^{i_{0}}, v_{1}^{i_{1}}, \ldots, v_{n-1}^{i_{n-1}}\right)\right)=v_{n}^{-1}\left(B P \wedge M\left(p^{i_{0}}, v_{1}^{i_{1}}, \ldots, v_{n-1}^{i_{n-1}}\right)\right) .
$$

The maps that build $B P \wedge M\left(p^{i_{0}}, v_{1}^{i_{1}}, \ldots, v_{n-1}^{i_{n-1}}\right)$ from $P(n)$ by cofibre sequences can all be chosen to be $B P$ module maps. Thus they will also build $v_{n}^{-1}(B P \wedge$ $\left.M\left(p^{i_{0}}, v_{1}^{i_{1}}, \ldots, v_{n-1}^{i_{n-1}}\right)\right)$ from $v_{n}^{-1} P(n)$. Thus

$$
\langle B P \wedge \operatorname{Tel}(n)\rangle \leq\left\langle v_{n}^{-1} P(n)\right\rangle=\langle K(n)\rangle .
$$

The latter equality comes from Theorem 2.1 of [Rav84].

The unit map of $v_{n}^{-1} P(n)$ factors through $v_{n}^{-1}\left(B P \wedge M\left(p^{i_{0}}, v_{1}^{i_{1}}, \ldots, v_{n-1}^{i_{n-1}}\right)\right)$, so we also have $\langle B P \wedge \operatorname{Tel}(n)\rangle \geq\langle K(n)\rangle$.

Corollary 1.10. $B P \wedge A(n)=0$, so that the natural map $L_{n}^{f} X \rightarrow L_{n} X$ is a BP equivalence. 
ProOF.

$$
\langle B P \wedge A(n)\rangle=\langle B P \wedge \operatorname{Tel}(n) \wedge A(n)\rangle=\langle K(n) \wedge A(n)\rangle=0 .
$$

COROLlary 1.11. Every BP-module spectrum with finite acyclics is Bousfield equivalent to a finite wedge of Morava K-theories.

Proof. Suppose $E$ is a $B P$-module spectrum with $\mathrm{FA}(E)=\mathcal{C}_{n+1}$. Since $E$ is a $B P$ module spectrum, $E$ is a retract of $B P \wedge E$, so

$$
\langle E\rangle=\langle B P \wedge E\rangle=\langle B P \wedge E \wedge \operatorname{Tel}(0)\rangle \vee \cdots \vee\langle B P \wedge E \wedge \operatorname{Tel}(n)\rangle .
$$

But $\langle B P \wedge E \wedge \operatorname{Tel}(n)\rangle=\langle K(n) \wedge E\rangle$. Since $K(n)$ is a field spectrum, we have that $\langle K(n) \wedge E\rangle$ is either 0 or $\langle K(n)\rangle$.

A particularly good kind of $B P$-module spectrum is a Landweber exact spectrum [Land]. Recall that $E$ is Landweber exact if the natural map

$$
B P_{*}(X) \otimes_{B P_{*}} E_{*} \rightarrow E_{*}(X)
$$

is an isomorphism. The most common examples are $E(n)$ and elliptic cohomology. Call $E v_{n}$-periodic if $v_{n} \in B P_{*}$ maps to a unit in $E_{*} /\left(p, v_{1}, \ldots, v_{n-1}\right)$.

COROLlary 1.12. If $E$ is a $v_{n}$-periodic Landweber exact spectrum, then

$$
\langle E\rangle=\langle E(n)\rangle=\langle K(0) \vee \cdots \vee K(n)\rangle \text {. }
$$

Proof. Recall that if $E$ is $v_{n}$-periodic and Landweber exact then $v_{j}$ is not a zero-divisor $\bmod \left(p, v_{1}, \ldots, v_{j-1}\right)$ for $j<n$, and $v_{n}$ is a unit $\bmod \left(p, v_{1}, \ldots, v_{n-1}\right)$ [Land]. It suffices to show that $E \wedge K(j) \neq 0$ for $j \leq n$, and that $E \wedge F(n+1)=$ 0 . Since $E$ is a $B P$-module spectrum, $\langle E \wedge K(j)\rangle=\langle E \wedge T e l(j)\rangle$, it suffices to show that $E \wedge v_{j}^{-1} M\left(p^{i_{0}}, v_{1}^{i_{1}}, \ldots, v_{n-1}^{i_{j-1}}\right) \neq 0$. But the homotopy of $E \wedge v_{j}^{-1} M\left(p^{i_{0}}, v_{1}^{i_{1}}, \ldots, v_{n-1}^{i_{j-1}}\right)$ is $v_{j}^{-1} E_{*} /\left(p^{i_{0}}, v_{1}^{i_{1}}, \ldots, v_{j-1}^{i_{j-1}}\right)$, which is not 0 by Landweber exactness.

Similarly, the homotopy of $E \wedge M\left(p^{i_{0}}, v_{1}^{i_{1}}, \ldots, v_{n}^{i_{n}}\right)$ is $E_{*} /\left(p^{i_{0}}, v_{1}^{i_{1}}, \ldots, v_{n}^{i_{n}}\right)$. We know that $v_{n}$ is a unit $\bmod \left(p, v_{1}, \ldots, v_{n-1}\right)$, and it follows that $v_{n}$ is also a unit $\bmod \left(p^{i_{0}}, v_{1}^{i_{1}}, \ldots, v_{n-1}^{i_{n-1}}\right)$, so the homotopy is 0 .

\section{Localizations with respect to finite spectra}

In this section we consider what localization with respect to a finite spectrum looks like. We also determine the $K(n)$-localization of $B P$. All of the results in this section are known to Hopkins and possibly others. Special cases of some of these results have appeared in $[\mathbf{M S}]$. 
We have already used the $M\left(p^{i_{0}}, v_{1}^{i_{1}}, \ldots, v_{n-1}^{i_{n-1}}\right)$ in the previous section. We need them again here, and we need to know that they exist for sufficiently large $\left(i_{0}, \ldots, i_{n}\right)$. Furthermore, there are natural maps

$$
M\left(p^{j_{0}}, v_{1}^{j_{1}}, \ldots, v_{n-1}^{j_{n-1}}\right) \rightarrow M\left(p^{i_{0}}, v_{1}^{i_{1}}, \ldots, v_{n-1}^{i_{n-1}}\right)
$$

for $j_{k}$ sufficiently large compared to $i_{k}$, which induce the evident map on $B P$ homology. Notice that these maps fix the bottom cell.

The following result says that localization with respect to $F(n)$ is completion at $p, v_{1}, \ldots, v_{n-1}$.

Theorem 2.1. For arbitrary $X$, the map $X \rightarrow \varliminf_{(}\left(X \wedge M\left(p^{i_{0}}, v_{1}^{i_{1}}, \ldots, v_{n-1}^{i_{n-1}}\right)\right)$ induced by inclusion of the bottom cell is $F(n)$-localization.

Proof. First we verify that the right-hand side is $F(n)$-local. Suppose $Z$ is $F(n)$-acyclic. Then

$$
\left[Z, X \wedge M\left(p^{i_{0}}, v_{1}^{i_{1}}, \ldots, v_{n-1}^{i_{n-1}}\right)\right]=\left[Z \wedge D M\left(p^{i_{0}}, v_{1}^{i_{1}}, \ldots, v_{n-1}^{i_{n-1}}\right), X\right],
$$

where $D Y$ denotes the Spanier-Whitehead dual of $Y$. Since

$$
K(i)_{*}(D Y)=K(i)^{*}(Y)=\operatorname{Hom}_{K(i)_{*}}\left(K(i)_{*}(Y), K(i)_{*}\right),
$$

$D M\left(p^{i_{0}}, v_{1}^{i_{1}}, \ldots, v_{n-1}^{i_{n-1}}\right)$ also has type $n$. Thus

$$
Z \wedge D M\left(p^{i_{0}}, v_{1}^{i_{1}}, \ldots, v_{n-1}^{i_{n-1}}\right)=0,
$$

so $X \wedge M\left(p^{i_{0}}, v_{1}^{i_{1}}, \ldots, v_{n-1}^{i_{n-1}}\right)$ is $F(n)$-local. Then the inverse limit of $X \wedge$ $M\left(p^{i_{0}}, v_{1}^{i_{1}}, \ldots, v_{n-1}^{i_{n-1}}\right)$ is also $F(n)$-local.

Now we must check that the map is an $F(n)$-isomorphism. By SpanierWhitehead duality, it suffices to show that

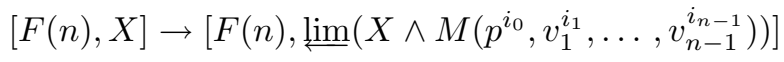

is an isomorphism. We have an exact sequence

$$
\begin{gathered}
\varliminf^{1}\left[F(n), X \wedge M\left(p^{i_{0}}, v_{1}^{i_{1}}, \ldots, v_{n-1}^{i_{n-1}}\right)\right] \hookrightarrow\left[F(n), \varliminf\left(X \wedge M\left(p^{i_{0}}, v_{1}^{i_{1}}, \ldots, v_{n-1}^{i_{n-1}}\right)\right)\right] \\
\rightarrow \varliminf^{\lim }\left[F(n), X \wedge M\left(p^{i_{0}}, v_{1}^{i_{1}}, \ldots, v_{n-1}^{i_{n-1}}\right)\right] \rightarrow 0 .
\end{gathered}
$$

There is a dimension shift on the $\varliminf^{1}$ term, but we will show it is 0 so that will not matter.

So we need to investigate $\left[F(n), X \wedge M\left(p^{i_{0}}, v_{1}^{i_{1}}, \ldots, v_{n-1}^{i_{n-1}}\right)\right]$, or equivalently, $\left[F(n) \wedge D M\left(p^{i_{0}}, v_{1}^{i_{1}}, \ldots, v_{n-1}^{i_{n-1}}\right), X\right]$. Note that $D M\left(p^{i_{0}}, v_{1}^{i_{1}}, \ldots, v_{n-1}^{i_{n-1}}\right)$ is just a desuspension of $M\left(p^{i_{0}}, v_{1}^{i_{1}}, \ldots, v_{n-1}^{i_{n-1}}\right)$, so that the top cell is in degree 0 . (This is easy to see from the construction of the $M\left(p^{i_{0}}, v_{1}^{i_{1}}, \ldots, v_{n-1}^{i_{n-1}}\right)$.) Also note that if $X$ is type $n, X \wedge M\left(p^{i_{0}}, v_{1}^{i_{1}}, \ldots, v_{n-1}^{i_{n-1}}\right)$ is a wedge of copies of $X$, for large enough indices $\left(i_{0}, \ldots, i_{n-1}\right)$. Indeed, at each stage of the construction of $M\left(p^{i_{0}}, v_{1}^{i_{1}}, \ldots, v_{n-1}^{i_{n-1}}\right)$, one takes the cofiber of a $v_{j}$ self map on 
$M\left(p^{i_{0}}, v_{1}^{i_{1}}, \ldots, v_{n-1}^{i_{j-1}}\right)$. Since $X$ is type $n$, that $v_{j}$ self map must be nilpotent on $X \wedge M\left(p^{i_{0}}, v_{1}^{i_{1}}, \ldots, v_{n-1}^{i_{j-1}}\right)$, so that if we take large enough indices, it will be null.

Thus $\left[F(n), X \wedge M\left(p^{i_{0}}, v_{1}^{i_{1}}, \ldots, v_{n-1}^{i_{n-1}}\right)\right]$ is a direct sum of copies of $[F(n), X]$ in dimensions corresponding to the cells of $D M\left(p^{i_{0}}, v_{1}^{i_{1}}, \ldots, v_{n-1}^{i_{n-1}}\right)$. The maps in the inverse system are all multiplication by a $v_{j}$ to some power, except on the top cell, which is fixed. So they are nilpotent, and for large enough indices will be 0 . Hence $\varliminf_{0}\left[F(n), X \wedge M\left(p^{i_{0}}, v_{1}^{i_{1}}, \ldots, v_{n-1}^{i_{n-1}}\right)\right]=[F(n), X]$ as required. Furthermore, the system is Mittag-Leffler, so the $\varliminf^{1}$ term vanishes as well.

Corollary 2.2 .

$$
L_{F(n) \wedge E} X=L_{F(n)} L_{E} X .
$$

Proof. The map $X \rightarrow L_{F(n)} L_{E} X$ is an $F(n) \wedge E$-isomorphism, so it suffices to show that $L_{F(n)} L_{E} X$ is $F(n) \wedge E$-local. Since

$$
L_{F(n)} L_{E} X=\varliminf_{(i m}\left(L_{E} X \wedge M\left(p^{i_{0}}, v_{1}^{i_{1}}, \ldots, v_{n-1}^{i_{n-1}}\right)\right),
$$

it will suffice to show that $L_{E} X \wedge M\left(p^{i_{0}}, v_{1}^{i_{1}}, \ldots, v_{n-1}^{i_{n-1}}\right)$ is $F(n) \wedge E$-local. Suppose $Z$ is $F(n) \wedge E$-acyclic, and consider

$$
\left[Z, L_{E} X \wedge M\left(p^{i_{0}}, v_{1}^{i_{1}}, \ldots, v_{n-1}^{i_{n-1}}\right)\right]=\left[Z \wedge D M\left(p^{i_{0}}, v_{1}^{i_{1}}, \ldots, v_{n-1}^{i_{n-1}}\right), L_{E} X\right] .
$$

$D M\left(p^{i_{0}}, v_{1}^{i_{1}}, \ldots, v_{n-1}^{i_{n-1}}\right)$ is type $n$, so $Z \wedge D M\left(p^{i_{0}}, v_{1}^{i_{1}}, \ldots, v_{n-1}^{i_{n-1}}\right)$ is $E$-acyclic, since $Z$ is $F(n) \wedge E$-acyclic. Thus this group is 0 as required.

Note that if $X$ is finite,

$$
\begin{gathered}
L_{F(n)} L_{E} X=\varliminf\left(L_{E} X \wedge M\left(p^{i_{0}}, v_{1}^{i_{1}}, \ldots, v_{n-1}^{i_{n-1}}\right)\right) \\
=\varliminf\left(X \wedge L_{E} M\left(p^{i_{0}}, v_{1}^{i_{1}}, \ldots, v_{n-1}^{i_{n-1}}\right)\right) .
\end{gathered}
$$

In particular, recalling from $[\mathbf{M S}]$ that

$$
L_{\operatorname{Tel}(n)} M\left(p^{i_{0}}, v_{1}^{i_{1}}, \ldots, v_{n-1}^{i_{n-1}}\right)=\operatorname{Tel}\left(M\left(p^{i_{0}}, v_{1}^{i_{1}}, \ldots, v_{n-1}^{i_{n-1}}\right)\right),
$$

and taking $E=\operatorname{Tel}(0) \vee \cdots \vee \operatorname{Tel}(n)$, we recover their result that

$$
L_{\operatorname{Tel}(n)} S^{0}=\varliminf\left(\operatorname{Tel}\left(M\left(p^{i_{0}}, v_{1}^{i_{1}}, \ldots, v_{n-1}^{i_{n-1}}\right)\right)\right) .
$$

We can use a similar argument to calculate $L_{K(n)} B P$.

LEMma 2.3.

$$
L_{K(n)} B P=L_{F(n)}\left(v_{n}^{-1} B P\right)=\varliminf_{n}\left(v_{n}^{-1} B P \wedge M\left(p^{i_{0}}, v_{1}^{i_{1}}, \ldots, v_{n-1}^{i_{n-1}}\right)\right) .
$$

Proof. Note that $v_{n}^{-1} B P$ is Landweber exact and $v_{n}$ periodic, so has Bousfield class $\langle K(0) \vee \cdots \vee K(n)\rangle$. As a ring spectrum, it is self-local, so

$$
L_{n}\left(v_{n}^{-1} B P\right)=v_{n}^{-1} B P .
$$

Thus

$$
L_{K(n)}\left(v_{n}^{-1} B P\right)=L_{F(n)} L_{n}\left(v_{n}^{-1} B P\right)=L_{F(n)}\left(v_{n}^{-1} B P\right) .
$$


So it suffices to show that $B P \rightarrow v_{n}^{-1} B P$ is a $K(n)$-isomorphism, or equivalently that $B P \stackrel{\times v_{n}}{\longrightarrow} B P$ is a $K(n)$-isomorphism. (We have left out the evident suspension). Since $K(n)$ is a field spectrum and so has a Kunneth isomorphism, it will suffice to show that

$$
B P \wedge M\left(p^{i_{0}}, v_{1}^{i_{1}}, \ldots, v_{n-1}^{i_{n-1}}\right) \stackrel{\times v_{n}}{\longrightarrow} B P \wedge M\left(p^{i_{0}}, v_{1}^{i_{1}}, \ldots, v_{n-1}^{i_{n-1}}\right)
$$

is a $K(n)$-isomorphism.

Note that $\times v_{n}$ induces multiplication by $\eta_{R} v_{n}$ on $B P_{*} B P$ or $K(n)_{*} B P$. Here $\eta_{R}$ is the right unit, discussed in [Rav86], where it is shown that

$$
\eta_{R} v_{n} \equiv v_{n} \bmod \left(p, v_{1}, \ldots, v_{n-1}\right) .
$$

Thus, $\times v_{n}$ is an isomorphism on $K(n)_{*} P(n)$. But $B P \wedge M\left(p^{i_{0}}, v_{1}^{i_{1}}, \ldots, v_{n-1}^{i_{n-1}}\right)$ can be built from $P(n)$ using cofiber sequences where the maps are $B P$-module maps. Thus $\times v_{n}$ is also an isomorphism on

$$
K(n)_{*}\left(B P \wedge M\left(p^{i_{0}}, v_{1}^{i_{1}}, \ldots, v_{n-1}^{i_{n-1}}\right)\right) .
$$

The homotopy of $L_{K(n)} B P$ is then easily calculated to be $\left(v_{n}^{-1} B P_{*}\right)_{I_{n}}$, the completion of $v_{n}^{-1} B P_{*}$ at the ideal $I_{n}=\left(p, v_{1}, \ldots, v_{n-1}\right)$. Note that $v_{n}$ is not a unit in $L_{n} B P$, but becomes one upon completion at $I_{n}$. In particular, one sees that $L_{K(n)} B P$ is Landweber exact, so we have

Corollary 2.4 .

$$
\left\langle L_{K(n)} S^{0}\right\rangle=\langle K(0) \vee \cdots \vee K(n)\rangle
$$

Proof. $\left\langle L_{K(n)} S^{0}\right\rangle \leq\left\langle L_{n} S^{0}\right\rangle$, sice $L_{K(n)} S^{0}$ is an $L_{n} S^{0}$ module spectrum. Since $L_{n}$ is smashing, $\left\langle L_{n} S^{0}\right\rangle=\langle K(0) \vee \cdots \vee K(n)\rangle$. On the other hand, $\mathrm{E}_{K(n)} B P$ is an $L_{K(n)} S^{0}$-module spectrum, and since $L_{K(n)} B P$ is Landweber exact,

$$
\left\langle L_{K(n)} B P\right\rangle=\langle K(0) \vee \cdots \vee K(n)\rangle .
$$

\section{Ring spectra without finite acyclics}

In this section we prove our $B P$-version of the chromatic splitting conjecture and use it to deduce that finite torsion spectra are local with respect to any infinite wedge of Morava K-theories. A corollary of this is that localization with respect to a ring spectrum that has no finite acyclics must be the identity functor or $p$-completion on finite complexes.

Recall that all spectra are $p$-local, and $X_{p}$ denotes the $p$-completion of $X$. Throughout this section $\left(n_{i}\right)$ will be an infinite increasing sequence of nonnegative integers. 
THEOREM 3.1. The natural map

$$
B P_{p} \rightarrow \prod_{i=1}^{\infty} L_{K\left(n_{i}\right)} B P_{p}
$$

is the inclusion of a wedge summand.

To prove this theorem, we use Brown-Comanetz duality. Recall that the Brown-Comanetz dual of a spectrum $X$ is the spectrum $I X$ which represents the functor $Y \rightarrow \operatorname{Hom}\left(\pi_{0}(X \wedge Y), \mathbf{Q} / \mathbf{Z}\right)$. In particular, if $X$ has finitely generated homotopy groups, then $I^{2} X=X_{p}$. Recall as well that a map $Y \rightarrow X$ is called f-phantom if, for all finite $Z$ and maps $Z \rightarrow Y$, the composite $Z \rightarrow Y \rightarrow X$ is null. Recall the following lemma, on page 66 of [Mar].

Lemma 3.2. For any spectrum $X$, any f-phantom map into $I X$ is null.

Let $F$ be the fibre of $B P_{p} \rightarrow \prod_{i=1}^{\infty} L_{K\left(n_{i}\right)} B P_{p}$. Since $B P_{p}=I(I(B P))$, we will have proved the theorem if we can show that the map $F \rightarrow B P_{p}$ is f-phantom.

First we remove the p-completion.

LEMma 3.3. Let $F^{\prime}$ be the fibre of

$$
B P \rightarrow \prod_{i=1}^{\infty} L_{K\left(n_{i}\right)} B P .
$$

If $F^{\prime} \rightarrow B P$ is f-phantom, then $F \rightarrow B P_{p}$ is null.

Proof of Lemma. Let $C$ be the fiber of $B P \rightarrow B P_{p}$. Then $C$ is a rational space, so $L_{K(n)} C=*$, and $L_{K(n)} B P=L_{K(n)}\left(B P_{p}\right)$ for $n>0$. Consider the following diagram.

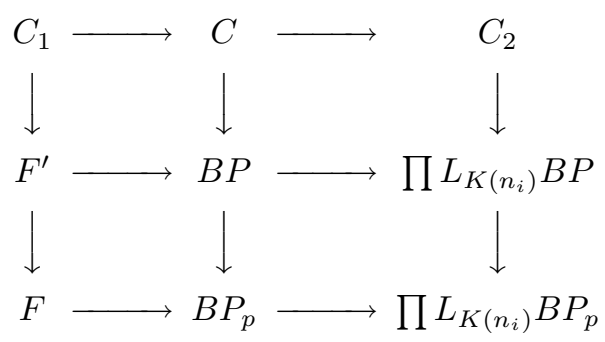

We consider two cases. If $L_{K(0)}$ appears in the product, then

$$
C_{2}=\operatorname{fiber}\left(L_{K(0)} B P \rightarrow L_{K(0)}\left(B P_{p}\right)\right)=C,
$$

so that $F=F^{\prime}$. Then if $F^{\prime} \rightarrow B P$ is f-phantom, so is $F \rightarrow B P_{p}$, and so it is null.

On the other hand, if $L_{K(0)}$ does not appear in the product, then $C_{2}=0$, and we have a cofiber sequence

$$
F^{\prime} \rightarrow F \rightarrow \Sigma C
$$


If $F^{\prime} \rightarrow B P$ is f-phantom, then $F^{\prime} \rightarrow B P \rightarrow B P_{p}$ is null, so $F \rightarrow B P_{p}$ factors through $\Sigma C$. But $C$ is $M(p)$-acyclic and $B P_{p}$ is $M(p)$-local, so the map must be null.

So to complete the proof of the theorem, it will suffice to prove:

Lemma 3.4. If $X$ is finite, the map $B P^{*}(X) \rightarrow\left(L_{K(n)} B P\right)^{*}(X)$ is injective for large $n$.

Proof. By using Spanier-Whitehead duality, it suffices to prove the lemma in homology rather than cohomology. Recall from the preceeding sections that $L_{K(n)} B P_{*}=\left(v_{n}^{-1} B P_{*}\right)_{I_{n}}$, where $I_{n}=\left(p, v_{1}, \ldots v_{n-1}\right)$ as usual. Note that $L_{K(n)} B P$ clearly satisfies the hypotheses of the Landweber exact functor theorem, so that

$$
\left(L_{K(n)} B P\right)_{*}(X)=B P_{*}(X) \otimes_{B P_{*}} L_{K(n)} B P_{*} .
$$

The Landweber filtration theorem [Land] says that $B P_{*}(X)$ has a finite filtration by $B P_{*} B P$ subcomodules $M_{i}$ for $i=1, \ldots, m$, such that the quotient $M_{i+1} / M_{i}$ is isomorphic to $B P_{*} / I_{m_{i}}$ for some $m_{i}$. Choose $n$ larger than all the $m_{i}$. Then $B P_{*} / I_{m_{i}}$ injects into $B P_{*} / I_{m_{i}} \otimes_{B P_{*}}\left(v_{n}^{-1} B P_{*}\right)_{I_{n}}$. The proof of the Landweber exact functor theorem actually shows that $\left(v_{n}^{-1} B P_{*}\right)_{I_{n}}$ is flat in the category of $B P_{*} B P$ comodules which are finitely generated over $B P_{*}$. Now an easy induction on the $M_{i}$ using the 5-lemma completes the proof.

Corollary 3.5. $B P_{p}$ is local with respect to $E=\bigvee K\left(n_{i}\right)$ for any infinite sequence $\left(n_{i}\right)$ of integers. BP is E-local if and only if the sequence contains 0.

Proof. $L_{K\left(n_{i}\right)} B P_{p}$ is certainly $E$-local, and any product of local spectra is local. Thus $B P_{p}$ is a retract of a local space, so is local. We have the cofiber sequence $C \rightarrow B P \rightarrow B P_{p}$, where $C$ is rational. Thus, $B P$ is $E$-local if and only if $C$ is $E$-local if and only if $H \mathbf{Q}$ is $E$-local. This is true if and only if 0 is in the sequence.

It is natural to ask if the analogue of chromatic convergence holds. Define $X_{j}=L_{K\left(n_{0}\right) \vee \ldots \vee K\left(n_{j}\right)} B P_{p}$. One would then ask if $B P_{p}$ is the inverse limit $X$ of the $X_{j}$. I don't know the answer to this question. Note though that the map from $B P_{p} \rightarrow \prod L_{K\left(n_{i}\right)} B P_{p}$ factors through $X$, so that $B P_{p}$ is a retract of $X$.

THEOREM 3.6. Suppose $R$ is a ring spectrum with no finite acyclics. If $H \mathbf{Q} \wedge$ $R \neq *$, then $L_{R} X=X$ for all finite $X$. If $H \mathbf{Q} \wedge R=*$, then $L_{R} X=X_{p}$ for all finite $X$.

First we show

Lemma 3.7. Suppose $E$ is any spectrum such that $L_{E} X=X$ for some finite $X$. Then if If $H \mathbf{Q} \wedge E \neq *$, then $L_{E} X=X$ for all finite $X$. If $H \mathbf{Q} \wedge E=*$, then $L_{E} X=X_{p}$ for all finite $X$. 
Proof. Consider the class $\mathcal{C}$ of all finite $X$ that are local with respect to $E$. It is easy to see that $\mathcal{C}$ is closed under retracts, suspensions, and cofibrations. It is nonempty by hypothesis, so it must be a $\mathcal{C}_{n}$ for some $n$. Suppose $n>1$, and let $X$ be a space of type $n-1$. Then $X$ has a $v_{n-1}$-self map $f$, which must be of positive degree $d$. In the cofiber sequence $\Sigma^{d} X \rightarrow X \rightarrow Y, Y$ has type $n$ so is $E$-local. Thus, if $Z$ is $E$-acyclic, any map $Z \stackrel{g}{\rightarrow} X$ factors through $\Sigma^{d} X$. Repeating this process, we find that $g$ factors through the inverse limit of the $\Sigma^{k d} Y$, which is null. Thus $X$ is $E$-local, which is a contradiction.

Thus $\mathcal{C} \supseteq \mathcal{C}_{1}$. In particular, the Moore space $M(p)$ is $E$-local. Consider the cofiber sequence $S_{p}^{0} \stackrel{\times p}{\rightarrow} S_{p}^{0} \rightarrow M(p)$. Again, if $Z$ is $E$-acyclic, any map $Z \rightarrow S_{p}^{0}$ factors through the inverse limit of the times $p$ map on the $p$-complete sphere, which is null. So $S_{p}^{0}$ is E-local.

Now consider the cofibre sequence

$$
F \rightarrow S^{0} \rightarrow S_{p}^{0}
$$

$F$ is a rational space, so it is either $E$-acyclic or $E$-local according to whether $E \wedge H \mathbf{Q}$ is trivial or not. Localizing the cofibre sequence at $E$ completes the proof of the lemma.

Proof of THEOREM. Thus to prove the theorem, we only need to show that some finite $X$ is $R$-local. A corollary of the nilpotence theorem [Hop] tells us that any ring spectrum must be detected by one of the $K(n)$, for $0 \leq n \leq \infty$. If $R$ is detected by $K(\infty)=H \mathbf{F}_{p}$ then the Bousfield class of $R$ is at least as big as that of $H \mathbf{F}_{p}$. Since the Moore space $M(p)$ is $H \mathbf{F}_{p}$-local, it is also $R$-local, and we are done.

So suppose that $R \wedge H \mathbf{F}_{p}$ is null. I claim that $R \wedge K(n)$ must then be nonzero for infinitely many $n<\infty$. Indeed, for all $n$, there is a ring spectrum $Y_{n}$ of type $n$. (see [Dev] for specific examples). Then $R \wedge Y_{n}$ is also a ring spectrum, which is nonzero since $R$ has no finite acyclics. It is not detected by any $K(i)$ with $i<n$ or $i=\infty$, so it must be detected by some $K(i)$ for $i \geq n$.

This means by [Rav84, Thm 2.1] that the Bousfield class of $R$ is as least as big as that of some infinite wedge of Morava K-theories. Thus it will suffice to show that $M(p)$ is local with respect to such a wedge, for then it will be $R$-local as well. To do this we follow the argument of [Rav84, Thm 4.4]. We know already that $B P_{p}$ is local. It follows that any locally finite wedge of suspensions of $B P \wedge M(p)$ is local. We then use the Adams tower based on $B P$ homology to write $M(p)$ as an inverse limit of spaces $K_{s}$ of the form $B P \wedge \overline{B P}^{\wedge s} \wedge M(p)$. Here $\overline{B P}$ is the fiber of the unit map $S^{0} \rightarrow B P$. Since $B P \wedge \overline{B P}$ is a locally finite wedge of suspensions of $B P$, each $K_{s}$ is local. Then $M(p)$, as the inverse limit of local spectra, is also local.

Corollary 3.5 and Theorem 3.6 can be used to show that some mapping groups are 0. For example, they imply that $\left[B P\langle n\rangle, B P_{p}\right]=0$ and $\left[B P\langle n\rangle, X_{p}\right]=0$, where $X$ is finite. Indeed, $B P\langle n\rangle$ has no $K(i)$ homology for $i>n$. 
Recall the problem of Bousfield, mentioned in Section 1, which asks for a classification of smashing localization functors.

COROLlary 3.8. If the localization functor $L_{E}$ is smashing and $E$ has no finite acyclics, then $L_{E}$ is the identity functor.

Proof. If $L_{E}$ is smashing, then $\langle E\rangle=\left\langle L_{E} S^{0}\right\rangle$, which is a ring spectrum. Since $E$ has no finite acyclics, neither does $L_{E} S^{0}$. So the proceeding theorem tells us that $L_{E} S^{0}$, which is $L_{L_{E}} S^{0} S^{0}$, is either $S^{0}$ or $S_{p}^{0}$. But $S_{p}^{0}$ has the same Bousfield class as the sphere itself. Indeed, suppose $S_{p}^{0} \wedge X$ is zero. Then, using the cofibre sequence

$$
F \rightarrow S^{0} \rightarrow S_{p}^{0}
$$

we find that $X$ is a rational space. But $S_{p}^{0} \wedge H \mathbf{Q}$ is not zero, so $S_{p}^{0} \wedge X$ can't be either. Thus $\langle E\rangle=\left\langle L_{E} S^{0}\right\rangle=\left\langle S^{0}\right\rangle$, as required.

This also proves the following conjecture in the case that $E$ is a ring spectrum with no finite acyclics. Hopkins and possibly others have made this conjecture independently.

CONJECTURE 3.9. If $E$ is arbitrary, then $L_{E} S^{0}$ is smashing.

This brings us to the question of localization with respect to an arbitrary spectrum with no finite acyclics. I make the following conjecture.

CONJECTURE 3.10. If $E$ has no finite acyclics, then $L_{E} S^{0}$ is either the sphere itself or $S_{p}^{0}$.

Our method above relied on showing that $B P_{p}$ is $E$-local. This will certainly not be true in general. There are $E$ with no finite acyclics such that $B P \wedge E$ is zero. An example of such a spectrum is $I S^{0}$, the Brown-Comanetz dual of the sphere. It is a consequence of sections 2 and 3 of [Rav84] that $B P \wedge I S^{0}=0$. However, torsion finite spectra are local with respect to $I S^{0}$. In fact $I^{2} X$ is always $I X$-local, since

$$
\left[Z, I^{2} X\right]=\left[Z, F\left(I X, I S^{0}\right)\right]=\left[Z \wedge I X, I S^{0}\right] .
$$

So $S_{p}^{0}=I^{2} S^{0}$ is local with respect to $I S^{0}$.

\section{The chromatic splitting conjecture}

In this section, we describe Hopkins' chromatic splitting conjecture and deduce some corollaries of it. The conjecture is concerned with the fibre of the map $L_{n} S^{0} \rightarrow L_{K(n)} S^{0}$. The following lemma is a generalization of a lemma of Hopkins.

Lemma 4.1. Suppose E, $F$ are spectra such that $F \wedge L_{E} S^{0}$ is null. Then for arbitrary $X$, the fibre of the natural map $L_{E \vee F} X \rightarrow L_{F} X$ is the function spectrum $F\left(L_{E} S^{0}, L_{E \vee F} X\right)$. 
Proof. Let $Y$ denote the fibre. Then $Y$ is $E \vee F$ local and $F$ acyclic. We claim that $Y$ is therefore $E$ local. Consider the map $Y \rightarrow L_{E} Y$. This is an $E$ isomorphism, and $F_{*} Y=0$. Now $L_{E} Y$ is an $L_{E} S^{0}$ module spectrum, so since $F \wedge L_{E} S^{0}$ is null, so is $F \wedge L_{E} Y$. Thus the map $Y \rightarrow L_{E} Y$ is an $E \vee F$ isomorphism. Since both sides are $E \vee F$ local, it is therefore an equivalence, so $Y$ is $E$ local.

To show that $Y$ is $F\left(L_{E} S^{0}, L_{E \vee F} X\right)$, it will suffice to show that $Y$ has the same universal property, i.e. that

$$
[Z, Y]=\left[Z \wedge L_{E} S^{0}, L_{E \vee F} X\right] .
$$

Since $Y$ is $E$ local, and the natural map $Z \rightarrow Z \wedge L_{E} S^{0}$ is an $E$ isomorphism, we have $[Z, Y]=\left[Z \wedge L_{E} S^{0}, Y\right]$. Since $L_{E} S^{0}$ is $F$ acyclic, so is $Z \wedge L_{E} S^{0}$. Applying $[Z$,$] to the cofibre sequence$

$$
Y \rightarrow L_{E \vee F} X \rightarrow L_{F} X
$$

we see that $\left[Z \wedge L_{E} S^{0}, Y\right]=\left[Z \wedge L_{E} S^{0}, L_{E \vee F} X\right]$, as required.

The main example we are interested in here is the cofibre sequence

$$
F\left(L_{n-1} S^{0}, L_{n} X\right) \rightarrow L_{n} X \rightarrow L_{K(n)} X
$$

To describe the chromatic splitting conjecture, I must briefly describe some work of Hopkins-Ravenel and Hopkins-Miller based on Morava's philosophy. Unfortunately, little of this work has appeared. The idea is this: the Morava stabilizer group $S_{n}$ is essentially the group of automorphisms of the formal group law over $K(n)_{*}$. This is not quite true: it is actually the automorphisms of the same formal group law, but considered over the $\operatorname{ring} \mathbf{F}_{p^{n}}\left[u, u^{-1}\right]$. Here $u$ has degree -2 and is a $-\left(p^{n}-1\right)$-fold root of $v_{n}$. It is technically advantageous to use $u$ instead of $v_{n}$. The work of Lubin and Tate gives an action of $S_{n}$ on a complete ring whose residue field is $\mathbf{F}_{p^{n}}\left[u, u^{-1}\right]$. We take this ring to be the flat $E(n)_{*}$-module

$$
E_{n_{*}}=W\left(\mathbf{F}_{p^{n}}\right)\left[\left[u_{1}, \ldots, u_{n-1}\right]\right]\left[u, u^{-1}\right] .
$$

Here the $u_{i}$ have degree $0, u$ has degree -2 , and $W\left(\mathbf{F}_{p^{n}}\right)$ is the Witt vectors of the field with $p^{n}$ elements. The map

$$
E(n)_{*}=\mathbf{Z}_{(p)}\left[v_{1}, \ldots, v_{n}, v_{n}^{-1}\right] \rightarrow E_{n_{*}}
$$

takes $v_{i}$ to $u_{i} u^{1-p^{i}}$ and $v_{n}$ to $u^{1-p^{n}}$. The residue field of the complete local ring $E_{n_{*}}$ is then $\mathbf{F}_{p^{n}}\left[u, u^{-1}\right]$. Now given an element of $S_{n}$, it lifts to an isomorphism from the formal group $F$ over $E_{n_{*}}$ to a possibly different formal group $F^{\prime}$. The work of Lubin-Tate $[\mathbf{L T}]$ shows that there is a well-defined automorphism of the ring $E_{n_{*}}$ taking $F^{\prime}$ to a formal group law which is *-isomorphic to $F$, i.e. isomorphic by an isomorphism which reduces to the identity on the residue field $\mathbf{F}_{p^{n}}\left[u, u^{-1}\right]$. This gives a (continuous) action of $S_{n}$ on $E_{n_{*}}$. 
Now, $E_{n_{*}}$ is actually the homotopy of a spectrum $E_{n}$. In fact, $E_{n_{*}}$ is a flat $E(n)_{*}$-module, so one can simply tensor with it. In $[\mathbf{H M}]$ it is shown that $S_{n}$ actually acts on the spectrum $E_{n}$, in fact by $E_{\infty}$ maps. They show that the homotopy fixed point spectrum of this action is $L_{K(n)} S^{0}$. (Actually, one has to cope with the Galois group $\mathbf{Z} / n$ of the extension $W\left(\mathbf{F}_{p^{n}}\right)$ over $\mathbf{Z}_{p}$ as well.) There is then a homotopy fixed point set spectral sequence

$$
E_{2}=H^{*, *}\left(S_{n} ; E_{n_{*}}\right)^{\mathbf{Z} / n} \Longrightarrow \pi_{*}\left(L_{K(n)} S^{0}\right) .
$$

The group cohomology here must be taken to be continuous cohomology. This spectral sequence was known before the work of $[\mathbf{H M}]$ : I believe it is due to Hopkins-Ravenel, and a brief description of it appears in [HMS]. It collapses and there are no extensions when the prime $p$ is large with respect to $n$.

Now, consider the inclusion

$$
W\left(\mathbf{F}_{p^{n}}\right) \rightarrow E_{n, 0} .
$$

This is a map of $S_{n}$-modules, where $S_{n}$ acts trivially on $W\left(\mathbf{F}_{p^{n}}\right)$, so induces

$$
H^{*}\left(S_{n} ; W\left(\mathbf{F}_{p^{n}}\right)\right) \rightarrow H^{*}\left(S_{n} ; E_{n, 0}\right) .
$$

(Here and below I always mean the $\mathbf{Z} / n$ invariants of cohomology groups.) Computations suggest that $H^{*}\left(S_{n} ; W\left(\mathbf{F}_{p^{n}}\right)\right)$ is, or at least contains, an exterior algebra on $n$ generators $x_{1}, \ldots, x_{n}$. Of these, $x_{1}$ is most familiar: it is usually called $\zeta_{n}$. It arises from the determinant map $S_{n} \rightarrow \mathbf{Z}_{p}$, where we are thinking of $\mathbf{Z}_{p}$ as a subgroup of its own group of units. This is a crossed homomorphism with respect to the trivial action of $S_{n}$ on $Z_{p} \subseteq W\left(\mathbf{F}_{p^{n}}\right)$, so gives rise to a class in $H^{1}\left(S_{n} ; W\left(\mathbf{F}_{p^{n}}\right)\right)$.

I will describe Hopkins' chromatic splitting conjecture first in the case $n=2$ where it is simpler and also known to be true, at least for $p>3$. This is all due to Hopkins, and uses essentially the calculations of Shimomura-Yabe in $[\mathbf{S h}-\mathbf{Y}]$. In that case, $H^{*}\left(S_{2} ; W\left(\mathbf{F}_{p^{n}}\right)\right)$ is an exterior algebra on classes traditionally denoted $\zeta$ and $\rho$, in bidegrees $(1,0)$ and $(3,0)$. Both of these classes survive to homotopy classes

$$
\zeta: S_{p}^{-1} \rightarrow L_{K(2)} S^{0} \rho: S_{p}^{-3} \rightarrow L_{K(2)} S^{0} .
$$

Multiplication also gives us a class

$$
\zeta \rho: S_{p}^{-4} \rightarrow L_{K(2)} S^{0} .
$$

Compose these maps with the map

$$
L_{K(2)} S^{0} \rightarrow F\left(L_{1} S^{0}, L_{2} S_{p}^{0}\right) .
$$

This is $L_{1}$-local, so we get maps $\zeta, \rho$, and $\zeta \rho$ from $L_{1} S_{p}^{0}$ to $F\left(L_{1} S^{0}, L_{2} S_{p}^{0}\right)$ of degrees $-2,-4,-5$. But, strangely enough, $\rho$ and $\zeta \rho$ actually factor further through $L_{0} S_{p}^{0}$. Thus we get a map

$$
\Sigma^{-2} L_{1} S_{p}^{0} \vee \Sigma^{-4} L_{0} S_{p}^{0} \vee \Sigma^{-5} L_{0} S_{p}^{0} \rightarrow F\left(L_{1} S^{0}, L_{2} S_{p}^{0}\right) .
$$


This map is in fact a homotopy equivalence. This is the chromatic splitting conjecture for $n=2$. The general case is more complicated and is stated below.

Conjecture 4.2 (Hopkins' Chromatic Splitting Conjecture). Fix an integer $n \geq 1$.

(i) $H^{*}\left(S_{n} ; W\left(\mathbf{F}_{p^{n}}\right)\right)$ contains the exterior algebra $E\left(x_{1}, \ldots, x_{n}\right)$.

(ii) Each class of nonzero degree $x_{i_{1}} \cdots x_{i_{j}}$ in the exterior algebra survives to a homotopy class

$$
x_{i_{1}} \cdots x_{i_{j}}: S_{p}^{-2\left(\Sigma i_{k}\right)+j} \rightarrow L_{K(n)} S^{0} .
$$

(iii) The composite

$$
S_{p}^{-2\left(\Sigma i_{k}\right)+j} \stackrel{x_{i_{1}} \cdots x_{i_{j}}}{\rightarrow} L_{K(n)} S^{0} \rightarrow \Sigma F\left(L_{n-1} S^{0}, L_{n} S_{p}^{0}\right)
$$

factors through

$$
L_{n-\max i_{k}} S_{p}^{-2\left(\Sigma i_{k}\right)+j} .
$$

(iv) The maps above split $F\left(L_{n-1} S^{0}, L_{n} S_{p}^{0}\right)$ into $2^{n}-1$ summands.

(v) The cofibre sequence

$$
F\left(L_{n-1} S^{0}, L_{n} S_{p}^{0}\right) \rightarrow L_{n-1} S_{p}^{0} \rightarrow L_{n-1} L_{K(n)} S^{0}
$$

splits, so that

$$
L_{n-1} L_{K(n)} S^{0} \simeq L_{n-1} S_{p}^{0} \vee \Sigma F\left(L_{n-1} S^{0}, L_{n} S_{p}^{0}\right) .
$$

As mentioned above, this conjecture is known to be true for $n=1$ and for $n=2, p>3$. The only other thing known about this conjecture is that $x_{1}=\zeta_{n}$ always survives to give a homotopy class $[\mathbf{H M}]$. One would expect that part 1 should be possible to do, and that part 2 may be approachable using the techniques of $[\mathbf{H M}]$. To this author at least, part 3 is a complete mystery. It seems to be suggesting that there is some interesting relationship between the different $S_{n}$. Note that part 5 of the chromatic splitting conjecture has not really been tested yet. The summand $\Sigma^{-2} L_{n-1} S_{p}^{0}$ of $F\left(L_{n-1} S^{0}, L_{n} S_{p}^{0}\right)$ corresponding to $\zeta_{n}$ always maps trivially to $L_{n-1} S_{p}^{0}$ by construction. For $n=2$, the maps from the other 2 summands to $L_{1} S_{p}^{0}$ are trivial for dimensional reasons. For $n=3$ there are possible maps from the other summands to $L_{2} S_{p}^{0}$. It is also part 5 from which the striking corollaries below can be derived.

THEOREM 4.3. If the chromatic splitting conjecture is true, and if $f: X \rightarrow Y$ is a map between two finite spectra such that $L_{K(n)} f: L_{K(n)} X \rightarrow L_{K(n)} Y$ is null for infinitely many $n$, then $f$ is null.

ProOF. It suffices to show that $f: X \rightarrow Y_{p}$ is null. Note that $L_{K(n)} Y_{p}=$ $L_{K(n)} Y$ if $n>0$. We have the diagram 


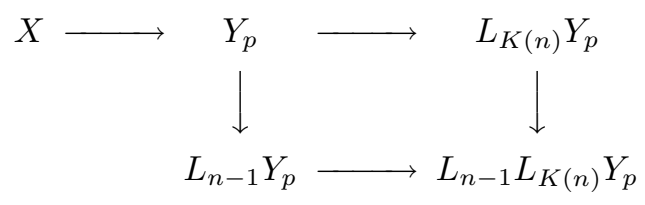

By the preceeding result, $L_{n-1} Y_{p}$ is a summand of $L_{n-1} L_{K(n)} Y_{p}$. Thus if $X \rightarrow L_{K(n)} Y_{p}$ is null, so is $X \rightarrow L_{n-1} Y_{p}$. The chromatic convergence theorem says that the tower $L_{n-1} Y$ is pro-isomorphic to the constant tower. It is easy to see that $L_{n-1} Y_{p}$ is also pro-isomorphic to the constant tower. Thus, since $X \rightarrow L_{n-1} Y_{p}$ is null for a cofinal sequence of $n$ 's, $X \rightarrow Y_{p}$ is null.

We can use the results in the previous section to prove that such a map must at least be null upon smashing with $B P$.

Proposition 4.4. If $f: X \rightarrow Y$ is a map between two finite spectra such that $L_{K(n)} f$ is null for infinitely many $n$, then the composite

$$
X \rightarrow Y \rightarrow B P \wedge Y
$$

is null. In particular, if $E$ is a BP-module spectrum, $E_{*}(f): E_{*}(X) \rightarrow E_{*}(Y)$ is zero.

Proof. First note that infinite products commute with smashing with finite spectra, by Spanier-Whitehead duality. Thus, $B P_{p} \wedge Y$ is a retract of $\prod L_{K\left(n_{i}\right)} B P_{p} \wedge Y$, for any infinite sequence $\left(n_{i}\right)$. Since the map $X \rightarrow Y \rightarrow$ $B P_{p} \wedge Y$ becomes null on localizaing with respect to $K(n)$ for infinitely many $n$, it is null. It follows from general facts about $p$-completions of spectra of finite type that the map

$$
X \rightarrow Y \rightarrow B P \wedge Y
$$

is null (see Chapter 9 of [Mar]). Smashing with $E$, we find that the composite

$$
E \wedge X \rightarrow E \wedge Y \rightarrow E \wedge B P \wedge Y
$$

is null. But if $E$ is a $B P$-module spectrum, then $E$ is a wedge summand of $E \wedge B P$, so in fact $E \wedge X \rightarrow E \wedge Y$ is null.

For several years, Hopkins has been saying that one does not need to reassemble the monochromatic parts of $X$ to recover the homotopy theory of finite spectra. The following corollary indicates a precise sense in which this is true.

COROLlary 4.5. If the chromatic splitting conjecture is true, then the natural map $X_{p} \rightarrow \prod L_{K\left(n_{i}\right)} X_{p}$ is the inclusion of a summand. In particular, if $Y$ is arbitrary, and $Y \rightarrow X$ is a map such that the composite $Y \rightarrow X \rightarrow L_{K(n)} X$ is null for infinitely many $n$, then $Y \rightarrow X \rightarrow X_{p}$ is null. 
Proof. By the preceeding theorem, the map $X_{p} \rightarrow \prod_{i} L_{K\left(n_{i}\right)} X_{p}$ is injective on maps from finite complexes. Thus, if $F$ denotes the fibre, the map $F \rightarrow X_{p}$ is $f$-phantom. Since there are no $f$-phantom maps to $X_{p}$, it is null.

\section{Appendix: The $p$-completion}

In this appendix, we investigate the consequences that the chromatic splitting conjecture would have on the structure of $\pi_{*} L_{n} S^{0}$. In particular, we show how to determine the divisible summands, and show that except for those summands and the free one in dimension $0, \pi_{*} L_{n} S^{0}$ is a direct sum of cyclic groups which have bounded torsion in each dimension.

The idea is that the adjoint properties of function spectra together with the fact that $L_{n}$ is smashing allow us to deduce the structure of $F\left(L_{i} S^{0}, L_{n} S_{p}^{0}\right)$ from just knowing it for $i=n-1$. Indeed, we have

$$
\begin{aligned}
F\left(L_{i-1} S^{0}, L_{n} S_{p}^{0}\right)= & F\left(L_{i-1}\left(L_{i} S^{0}\right), L_{n} S_{p}^{0}\right)=F\left(L_{i-1} S^{0} \wedge L_{i} S^{0}, L_{n} S_{p}^{0}\right) \\
& =F\left(L_{i-1} S^{0}, F\left(L_{i} S^{0}, L_{n} S_{p}^{0}\right)\right) .
\end{aligned}
$$

We illustrate the technique for $n=2$, where we have

$$
F\left(L_{1} S^{0}, L_{2} S_{p}^{0}\right)=\Sigma^{-2} L_{1} S_{p}^{0} \vee \Sigma^{-4} L_{0} S_{p}^{0} \vee \Sigma^{-5} L_{0} S_{p}^{0}
$$

Thus,

$$
F\left(L_{0} S^{0}, L_{2} S_{p}^{0}\right)=\Sigma^{-4} L_{0} S_{p}^{0} \vee \Sigma^{-4} L_{0} S_{p}^{0} \vee \Sigma^{-5} L_{0} S_{p}^{0} .
$$

As we will see below, this splitting reflects the three $\mathbf{Q} / \mathbf{Z}_{p}$ summands in $\pi_{*} L_{2} S^{0}$ as calculated by Shimomura-Yabe in $[\mathbf{S h}-\mathbf{Y}]$.

Proposition 5.1. Suppose the chromatic splitting conjecture is true. Then $F\left(L_{0} S^{0}, L_{n} S_{p}^{0}\right)$ splits into a wedge of $3^{n-1}$ copies of $H \mathbf{Q}_{p}$, in dimensions ranging from $-2 n$ to $-n^{2}-1$, with $2^{n-1}$ copies in dimension $-2 n$.

It is of course possible to explicitly calculate the locations of these summands for any specific $n$. For example, for $n=3$, there are 4 summands in dimension $-6,3$ in dimension -7 , and 1 each in dimensions -9 and -10 .

Proof. We proceed by induction on $n$. Suppose that we know the result is true for all $k \leq n-1$. We have

$$
F\left(L_{0} S^{0}, L_{n} S_{p}^{0}\right)=F\left(L_{0} S^{0}, F\left(L_{n-1} S^{0}, L_{n} S_{p}^{0}\right)\right)
$$

as above. We know by the chromatic splitting conjecture that $F\left(L_{n-1} S^{0}, L_{n} S_{p}^{0}\right)$ splits into $2^{n}-1$ summands, with $L_{k} S_{p}^{0}$ occuring $2^{n-k-1}$ times. The highest dimensional occurence of $L_{k} S^{0}$ is in dimension $-2(n-k)$ (corresponding to $\left.x_{n-k} \in H^{2 k-1}\left(S_{n} ; W\left(\mathbf{F}_{p^{n}}\right)\right)\right)$ and the lowest dimensional occurence of $L_{k} S^{0}$ is in $-(n-k)^{2}-1$ (corresponding to $\left.x_{n-k} x_{n-k-1} \cdots x_{1}\right)$.

Thus the total number of summands in $F\left(L_{0} S^{0}, L_{n} S_{p}^{0}\right)$ is

$$
3^{n-2}+2 \times 3^{n-3}+\ldots+2^{n-2}+2^{n-1}=3^{n-1} .
$$


The only summands that can arise in dimension $-2 n$ arise from the highest dimensional occurence of $L_{k} S^{0}$. Thus there are

$$
2^{n-2}+2^{n-3}+\ldots+1=2^{n-1}
$$

of them. The lowest dimensional summand arises from choosing $k=0$ in the above paragraph, and occurs in dimension $-n^{2}-1$.

This proposition also explains why we need to complete the sphere in the chromatic splitting conjecture. If we did not, there would also be maps $\Sigma^{-1} H \mathbf{Q} \rightarrow$ $L_{n} S^{0}$ coming from the fiber of the natural map $L_{n} S^{0} \rightarrow L_{n} S_{p}^{0}$.

One certainly expects that $F\left(H \mathbf{Q}, L_{n} S_{p}^{0}\right)$ should correspond to the divisible summands in $\pi_{*} L_{n} S^{0}$. The rest of this section is devoted to proving that. Lemma 4.1 tells us that this function spectrum is the fibre of the map

$$
L_{n} S_{p}^{0} \rightarrow L_{K(1) \vee \cdots \vee K(n)} S_{p}^{0}
$$

Since

$$
L_{K(1) \vee \cdots \vee K(n)} S_{p}^{0}=L_{M(p)} L_{n} S_{p}^{0}
$$

we have a cofibre sequence

$$
F\left(L_{0} S^{0}, L_{n} S_{p}^{0}\right) \rightarrow L_{n} S_{p}^{0} \rightarrow\left(L_{n} S_{p}^{0}\right)_{p} .
$$

Now there are two things we need to do. First, we need to know something about how the homotopy groups of $X_{p}$ are related to the homotopy groups of $X$. One might like them to be the $p$-completions of the homotopy groups of $X$. This is false in general, but the following proposition says that they are close to being $p$-complete. That something like this proposition might be true was first suggested to me by Hal Sadofsky.

For an abelian group $G$, let $p^{\infty} G=\bigcap p^{n} G$.

Proposition 5.2. For arbitrary $X, Y,\left[Y, X_{p}\right]$ is a module over $\mathbf{Z}_{p}$, has no divisible summands, and $\left[Y, X_{p}\right] / p^{\infty}\left[Y, X_{p}\right]$ is the $p$-completion of $\left[Y, X_{p}\right]$.

Proof. We can assume $X=X_{p}$ and $Y=Y_{p}$. Then $Y$ is a module spectrum over $S_{p}^{0}$, so maps out of it are a module over $\pi_{0} S_{p}^{0}=\mathbf{Z}_{p}$. We will first show that $[Y, X]$ has no divisible summands. Consider the system of cofibre sequences whose $n$th and $n-1$ st terms are displayed below.

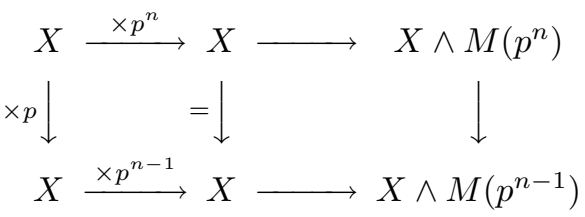

If $f \in[Y, X]$ generates a divisible summand, there are maps $f_{n} \in[Y, X]$ for all $n$, such that $p f_{n}=f_{n-1}$, where $f_{0}=f$. These will define a map into the inverse limit $Z=\varliminf_{\lim }(\times p: X \rightarrow X)$ of the left column in the above diagram. Now inverse limits do not behave very well in general, but the inverse limit of 
cofibre sequences is still a cofibre sequence, as we will prove below. Thus we get a cofibre sequence

$$
Z \rightarrow X \rightarrow \varliminf\left(X \wedge M\left(p^{n}\right)\right)=L_{M(p)} X .
$$

Since $X$ is already $M(p)$-local, $Z$ must be null. Since $f$ factors through $Z, f$ is null too.

Now we will show that the map

$$
[Y, X] \rightarrow[Y, X]_{p}=\varliminf_{[}[Y, X] / p^{n}[Y, X]
$$

is surjective. The proposition will then follow, since the kernel of the map $A \rightarrow A_{p}$ for abelian groups $A$ is always $p^{\infty} A$. Suppose $\left(f_{n}\right) \in[Y, X]_{p}$, so $f_{n} \in[Y, X] / p^{n}[Y, X]$. Let $A=[Y, X]$ and $B=[Y, \Sigma X]$, and denote the elements of $B$ killed by $\times p^{n}$ by $B\left(p^{n}\right)$. Then, using the cofibre sequence

$$
X \stackrel{\times p^{n}}{\rightarrow} X \stackrel{i}{\rightarrow} X \wedge M\left(p^{n}\right),
$$

we get a diagram of short exact sequences

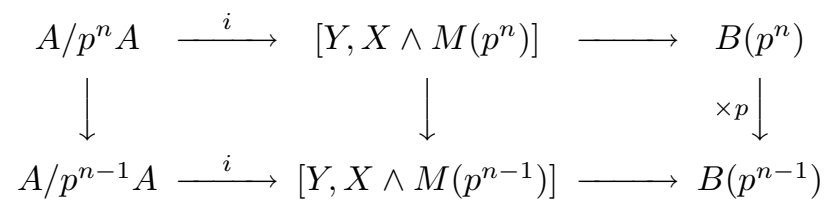

Since $\left(f_{n}\right)$ is a compatible sequence, so is $\left(i\left(f_{n}\right)\right)$, se we get an element of $\varliminf\left[Y, X \wedge M\left(p^{n}\right)\right]$. The map

$$
[Y, X]=\left[Y, \varliminf\left(X \wedge M\left(p^{n}\right)\right)\right] \rightarrow \varliminf_{[}\left[Y, X \wedge M\left(p^{n}\right)\right]
$$

is not an isomorphism in general, but it is always surjective. So we get a map $f \in[Y, X]$, and it is easy to see that $f$ maps to $\left(f_{n}\right) \in[Y, X]_{p}$.

This completes the proof of the proposition modulo the following lemma, which I learned from Hal Sadofsky.

LEMMA 5.3. The inverse limit of cofibre sequences is a cofibre sequence.

Proof. It is easy to see that products of cofibre sequences are cofibre sequences. Thus, given cofibre sequences

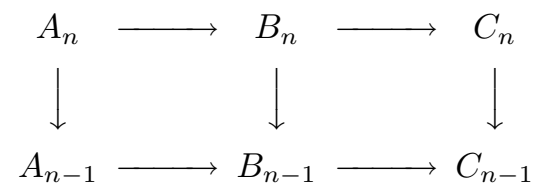

we get a diagram of cofibre sequences

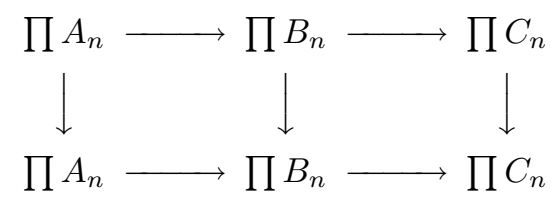


where the vertical arrows are the maps whose fibres are the inverse limits. Now it is not always true that the fibres in such a situation form a cofibre sequence, but it is true in this case since the map $\prod C_{n} \rightarrow \prod C_{n}$ is induced by the map $\prod B_{n} \rightarrow \prod B_{n}$.

Corollary 5.4. The kernel of the map

$$
[Y, X] \rightarrow\left[Y, X_{p}\right]
$$

is precisely the divisible summands in $[Y, X]$.

Proof. Any divisible summand in $[Y, X]$ must map to 0 in $\left[Y, X_{p}\right]$, by the proposition. To see the converse, note that we showed that the fibre of $X \rightarrow X_{p}$ is the rational spectrum $Z=\varliminf(\times p: X \rightarrow X)$. So $[Y, Z]$ is a divisible group, and thus its image in $[Y, X]$ is also divisible.

Now the second thing we need to do is to get some kind of control over the homotopy of $L_{n} S^{0}$.

Lemma 5.5. $\pi_{i} L_{n} S^{0}$ is a countable abelian group.

Proof. We will show this using the Adams-Novikov spectral sequence

$$
E_{2}^{s, t}=\operatorname{Ext}_{B P_{*} B P}^{s, s+i}\left(B P_{*}, B P_{*}\left(L_{n} S^{0}\right)\right) \Longrightarrow \pi_{i} L_{n} S^{0}
$$

This spectral sequence converges in a very strong sense, in that $E_{\infty}^{s, s+i}$ is 0 for large enough $s$ (and fixed $i$ ) [Rav92]. Thus, if $E_{\infty}^{s, s+i}$ is countable for all $s, t$, so is $\pi_{i} L_{n} S^{0}$. However, $E_{\infty}^{s, t}$ is a subquotient of $E_{2}^{s, s+i}$, so it will suffice to show that $E_{2}$ is countable in each bidegree.

One way to calculate the Ext groups of a $B P_{*} B P$ comodule $M$ is to use the cobar complex, made up out of

$$
\Omega^{s}(M)=M \otimes_{B P_{*}} B P_{*} B P \otimes_{B P_{*}} \cdots \otimes_{B P_{*}} B P_{*} B P
$$

where there are $s$ factors of $B P_{*} B P$. Note that $B P_{*} B P$ is countable in each degree. I claim that if $M, N$ are countable in each degree their tensor product will be too. Indeed, the degree $t$ part of their tensor product is a quotient of $\bigoplus M_{k} \otimes N_{t-k}$. The tensor product of two countable abelian groups is countable, as is the countable direct limit (or sum) of countable abelian groups. Thus, if $M$ is countable in each degree, so is $\Omega^{s} M$, and thus also $\operatorname{Ext}_{B P_{*} B P}^{s}\left(B P_{*}, M\right)$.

Thus it will suffice to show that $B P_{*}\left(L_{n} S^{0}\right)$ is countable in each degree. Since $L_{n}$ is smashing, $B P_{*}\left(L_{n} S^{0}\right)=\pi_{*}\left(L_{n} B P\right)$. This is calculated by Ravenel in [Rav84]. His result is that

$$
\pi_{*}\left(L_{n} B P\right)=B P_{*} \oplus \Sigma^{-n} N^{n+1}
$$

for $n \geq 1$, where $N_{n+1}$ is defined inductively by $N^{0}=B P_{*}$ and the short exact sequence

$$
0 \rightarrow N^{k} \rightarrow v_{k}^{-1} N^{k} \rightarrow N^{k+1} \rightarrow 0 .
$$


If $N^{k}$ is countable in each degree, so is $v_{k}^{-1} N^{k}$, as it is a direct limit of countable groups. So by induction, $N^{n+1}$ is countable in each degree, so is $B P_{*}\left(L_{n} S^{0}\right)$ and we are done.

Note that there is a sense in which countable torsion groups $A$ are completely classified (Ulm's Theorem [Kap]). This classification is complicated, however, because $p^{\infty} A$ may not be 0 . One certainly hopes that this complication does not arise in $L_{n} S^{0}$. We will see below that it does not if the chromatic splitting conjecture is true.

For the purposes of the theorem below, let $n_{k}$ denote the number of summands in $F\left(L_{0} S^{0}, L_{n} S_{p}^{0}\right)$ in dimension $k$.

THEOREM 5.6. Suppose the chromatic splitting conjecture is true. Then for all $k, \pi_{k} L_{n} S^{0}=D_{k} \oplus T_{k}$, where $D_{0}=\mathbf{Z}, D_{k}$ is a direct sum of $n_{k}$ copies of $\mathbf{Q} / \mathbf{Z}_{(p)}$, and $T_{k}$ is a bounded torsion group which is a countable direct sum of cyclic groups.

Proof. It is clear that $D_{0}=\mathbf{Z}$, since the composite $\pi_{0} S^{0} \rightarrow \pi_{0} L_{n} S^{0} \rightarrow$ $\pi_{0} L_{1} S^{0}$ is the identity. The rest of $\pi_{i} L_{n} S^{0}$ is all torsion. It suffices to prove the theorem for $X=L_{n} S_{p}^{0}$, which differs from $L_{n} S^{0}$ only in that $D_{0}=\mathbf{Z}_{p}$ instead of $\mathbf{Z}$.

So we have the cofibre sequence

$$
Y \rightarrow X \stackrel{f}{\rightarrow} X_{p}
$$

where $Y=F\left(L_{0} S^{0}, L_{n} S_{p}^{0}\right)$ is a finite wedge of suspensions of $H \mathbf{Q}_{p}$. We investigate the image of $f$ on homotopy. We have a short exact sequence

$$
0 \rightarrow A \rightarrow G \rightarrow H \rightarrow 0
$$

where $A=\pi_{k+1} X /$ (divisible summands) $=T_{k+1}$ is a countable torsion group, $G=\pi_{k+1} X_{p}$ is a $\mathbf{Z}_{p}$ module with no divisible summands whose $p$-completion is $G / p^{\infty} G$, and $H=\operatorname{im} f$ is torsion-free. This means that $A=\operatorname{Tor}(G)$, and that this is necessarily a short exact sequence of $\mathbf{Z}_{p}$ modules. There is an induced short exact sequence of $\mathbf{Z}_{p}$ modules

$$
0 \rightarrow A / p^{\infty} A \rightarrow G / p^{\infty} G \rightarrow H^{\prime} .
$$

The induced map of $\mathbf{Z}_{p}$ modules $H \rightarrow H^{\prime}$ is surjective, so since $H$ is a $\mathbf{Z}_{p}$ submodule of a finite direct sum of $\mathbf{Q}_{p}$ 's, $H^{\prime}$ can have at most only countable torsion.

Now, $B=A / p^{\infty} A$ is a countable torsion group, and $p^{\infty} B=0$. Thus, by Theorem 11 of [Kap], it must be a direct sum of cyclics

$$
B=\bigoplus_{i} \mathbf{Z} / p^{n_{i}} .
$$

Further, it is sitting inside the $p$-complete group $G / p^{\infty} G$. Therefore, its $p$ completion $B_{p}$ is also inside $G / p^{\infty} G$. If $B$ is unbounded torsion, one can see 
from the direct sum decomposition of $B$ that $B_{p} / B \subseteq H$ is uncountable, and in fact even the torsion of $B_{p} / B$ is uncountable. This is impossible, given the possibilities for $H^{\prime}$. Thus $B$ must be bounded torsion, say $p^{N} B=0$. But in that case, we have $p^{N} A \subseteq p^{\infty} A$, and so we can deduce that the times $p$ map from $p^{N} A$ to itself is surjective. This means $p^{N} A$ is divisible, and since $A$ has no divisible summands, it must be 0 . Therefore $A=T_{k+1}$ has bounded torsion, and is therefore a direct sum of cyclics, proving half of the proposition. But $A=\operatorname{Tor}(G)$, and a torsion subgroup which is bounded torsion always splits off. Thus $G=A \oplus H$. Thus $H=\operatorname{im} f$ can have no divisible summands. $H$ is therefore a free $\mathbf{Z}_{p}$ module.

If the rank of $H$ is less than $n_{k}$, then there is a $\mathbf{Q}_{p}$ summand in $\pi_{k} L_{n} S^{0}$. This summand survives to $\pi_{k} L_{0} S^{0}$, which is impossible. It follows that the image of $\pi_{k}(Y)$ in $\pi_{k} L_{n} S^{0}$ is a direct sum of $n_{k}$ copies of $\mathbf{Q} / \mathbf{Z}_{(p)}$, as required.

\section{REFERENCES}

[Bou79] A. K. Bousfield, The localization of spectra with respect to homology, Topology 18 (1979) 257-281.

[Bou92] A. K. Bousfield, Localization and periodicity in unstable homotopy theory, preprint (1992).

[Dev] E. S. Devinatz, Small ring spectra, Jour. Pure Appl. Alg., 81 (1992) $11-16$.

[DHS] E. Devinatz, M. Hopkins, and J. Smith, Nilpotence and stable homotopy theory I, Ann. Math. 128 (1988) 207-241.

[Hop] M. Hopkins, Global methods in homotopy theory, Proceedings of the 1985 LMS Symposium on Homotopy Theory, (J. D. S. Jones, E. Rees eds.), 1987, 73-96.

[HMS] M. Hopkins, M. Mahowald and H. Sadofsky, Constructions of elements in Picard groups, preprint (1992).

[HM] M. Hopkins and H. Miller, personal communication.

[HS] M. Hopkins and J. Smith, Nilpotence and stable homotopy theory II, preprint.

[Kap] I. Kaplansky, Infinite Abelian Groups, Univ. of Michigan Press (1954).

[Land] P. Landweber, Homological properties of comodules over $M U_{*}(M U)$ and $B P_{*}(B P)$, Amer. J. Math. 98 (1976) 591-610.

[LT] J. Lubin and J. Tate, Formal moduli for one-parameter Lie groups, Bull. Soc. Math. France 94 (1966) 49-60.

[MRS] M. Mahowald, D. Ravenel, and P. Shick, The v -periodic homotopy of a certain Thom complex, preprint (1992).

[MS] M. Mahowald and H. Sadofsky, $v_{n}$ telescopes and the Adams spectral sequence, preprint (1992).

[Mar] H. R. Margolis, Spectra and the Steenrod algebra, North-Holland (1983).

[Mil] H. R. Miller, Finite localizations, preprint (1992).

[Rav84] D. Ravenel, Localization with respect to certain periodic homology theories, Amer. J. Math. 106, (1984) 351-414. 
[Rav86] D. Ravenel, Complex cobordism and Stable Homotopy Groups of Spheres, Academic Press (1986).

[Rav92] D. Ravenel, Nilpotence and Periodicity in Stable Homotopy Theory, Princeton (1992).

[Rav92a] D. Ravenel, Life after the telescope conjecture, preprint (1992).

[Rav92b] D. Ravenel, A counterexample to the telescope conjecture, preprint (1992).

[Sh-Y] K. Shimomura and A. Yabe, The homotopy groups $\pi_{*}\left(L_{2} S^{0}\right)$, preprint (1992).

University OF KentuCKy, Lexington, KY

E-mail address: hovey@ms.uky.edu 